\title{
Some pseudovariety joins involving locally trivial semigroups and groups
}

\author{
José Carlos Costa*
}

September 27, 2001

\begin{abstract}
In this paper, we present the computation of some pseudovariety joins of the form $\mathcal{L} \mathbf{I} \vee \mathbf{H} \vee \mathbf{V}$ where $\mathcal{L} \mathbf{I}$ is the pseudovariety of locally trivial semigroups and $\mathbf{H}$ is any pseudovariety of groups. Similar results are obtained for the pseudovarieties $\mathbf{K}$, of semigroups in which idempotents are left zeros, and its dual $\mathbf{D}$, in the place of $\mathcal{L} \mathbf{I}$.
\end{abstract}

\section{Introduction}

For a pseudovariety $\mathbf{H}$ of groups, we denote by $\mathcal{D} \mathbf{R e H}$ the pseudovariety of all finite semigroups $S$ in which each regular $\mathcal{D}$-class is a rectangular group (i.e., a completely regular semigroup in which the idempotents form a subsemigroup) and all subgroups of $S$ lie in $\mathbf{H}$. We recall that $\mathcal{D} \mathbf{R e G}$ is usually denoted by $\mathcal{D} \mathbf{O}$, where $\mathbf{G}$ is the pseudovariety of all finite groups.

The purpose of this paper is the computation of some pseudovariety joins involving locally trivial semigroups and groups. To be more precise, if $\mathbf{H}$ is a pseudovariety of groups and $\mathbf{V}$ is a subpseudovariety of $(\mathbf{C R} m \mathbf{N}) \cap \mathcal{D} \mathbf{R e H}$, where $\mathbf{C R} m \mathbf{N}$ is the Mal'cev product of the pseudovarieties of completely regular semigroups and of nilpotent semigroups, we give a description of the joins of the form $\mathcal{L} \mathbf{I} \vee \mathbf{H} \vee \mathbf{V}$ in terms of a set of pseudoidentities defining $\mathbf{V}$. This work constitutes an extension of our paper [11], where we dealt with pseudovariety joins of the form $\mathcal{L} \mathbf{I} \vee \mathbf{V}$. In that paper, we introduced an operator $\mathcal{U}$ defined on the lattice of pseudovarieties as follows: if $\mathbf{V}$ is a pseudovariety defined by a set $\Sigma$ of pseudoidentities, then $\mathcal{U} \mathbf{V}$ is the pseudovariety defined by the pseudoidentities of the form $a^{\omega} b x c d^{\omega}=a^{\omega} b y c d^{\omega}$ where $x=y$ is an element of $\Sigma$. The pseudovariety $\mathcal{U} \mathbf{V}$ is a natural upper bound of the join $\mathcal{L} \mathbf{I} \vee \mathbf{V}$. In many cases the equality $\mathcal{L} \mathbf{I} \vee \mathbf{V}=\mathcal{U} \mathbf{V}$ holds. This is the case (see [11]) when $\mathbf{V}$ satisfies a certain cancellation property, which seams to be frequent: it is verified, for instance, by any pseudovariety of groups, by the pseudovariety Com of commutative semigroups and by the pseudovariety $\mathbf{J}$ of $\mathcal{J}$-trivial semigroups. However the equality $\mathcal{U} \mathbf{V}=\mathcal{L} \mathbf{I} \vee \mathbf{V}$ is not valid in general, as shown by the equality $\mathcal{L} \mathbf{I} \vee \mathbf{V}=\mathcal{U} \mathbf{V} \cap(\mathbf{C R} m \mathbf{N})$, verified by any subpseudovariety $\mathbf{V}$ of $\mathbf{C R} m \mathbf{N}$. Thus, for example, the pseudovariety $\mathcal{U} \mathbf{S l}$ constitutes a strict upper bound of $\mathcal{L} \mathbf{I} \vee \mathbf{S l}$, where $\mathbf{S l}$ is the pseudovariety of semilattices.

\footnotetext{
${ }^{*}$ Work supported, in part, by FCT through the Centro de Matemática da Universidade do Minho, and by the FCT and POCTI approved project POCTI/32817/MAT/2000 which is comparticipated by the European Community Fund FEDER.
} 
Here, we introduce a new operator which is related with the joins of the form $\mathcal{L} \mathbf{I} \vee$ $\mathbf{H} \vee \mathbf{V}$. Given a pseudovariety $\mathbf{V}$ defined by a set $\Sigma$ of pseudoidentities, we define $\mathcal{U}^{\omega} \mathbf{V}$ to be the pseudovariety defined by the pseudoidentities $\left(a^{\omega} b x c d^{\omega}\right)^{\omega}=\left(a^{\omega} b y c d^{\omega}\right)^{\omega}$ where $x=y$ is an element of $\Sigma$. The pseudovariety $\mathcal{U}^{\omega} \mathbf{V}$ is a natural upper bound of the joins $\mathcal{L} \mathbf{I} \vee \mathbf{H} \vee \mathbf{V}$ and it will be used to compute them when $\mathbf{V}$ is a subpseudovariety of $(\mathbf{C R} m \mathbf{N}) \cap \mathcal{D} \mathbf{R e H}$.

The problem of calculation of joins of pseudovarieties is in general very difficult and seams to depend greatly on the specific pseudovarieties involved. In fact, Albert, Baldinger and Rhodes [1] gave an example of two decidable pseudovarieties whose join is undecidable. This unexpected result has brought some attention and a new interest to the join operator and revealed the apparent impossibility of finding general results for doing computations. The join operator has received the attention of many authors and several calculations and answers to decision problems are known at the moment, but always involving specific pseudovarieties. In particular, the pseudovariety of groups is one of the most visited, from which the article of Almeida and Weil [6], where some difficult computations involving pseudovarieties of groups are obtained using elaborate techniques based on a study of profinite groups, constitutes a remarkable example. For other calculations, we must also cite the work of Almeida, Azevedo, Steinberg, Trotter, Volkov, Zeitoun and the author $[2,4,5,8,9,10,14,15,16,17]$. In [18] it is presented a survey on the subject.

\section{Preliminaries}

In this section, we will briefly recall some notions and results concerning the objects that we will be dealing with. We presuppose familiarity with the basic definitions of finite semigroup theory and implicit operations. For a comprehensive treatment of these subjects, the reader is referred to the books of Eilenberg [12], Pin [13] and Almeida [3].

For a pseudovariety $\mathbf{V}$, we let $\mathcal{L} \mathbf{V}\left(\operatorname{resp} . \mathcal{L}_{l} \mathbf{V}, \mathcal{L}_{r} \mathbf{V}\right)$ be the pseudovariety of all finite semigroups $S$ such that $e S e$ (resp. $e S, S e$ ) belongs to $\mathbf{V}$ for each idempotent $e$ of $S$. We notice that $\mathbf{K}=\mathcal{L}_{l} \mathbf{I}$ and $\mathbf{D}=\mathcal{L}_{r} \mathbf{I}$, where $\mathbf{I}$ denotes the trivial pseudovariety. We notice also that $\mathbf{C R} m \mathbf{N}=\mathcal{L}_{l} \mathbf{C R} \cap \mathcal{L}_{r} \mathbf{C R}$ since the pseudovarieties $\mathbf{C R} m \mathbf{N}$, $\mathcal{L}_{l} \mathbf{C R}$ and $\mathcal{L}_{r} \mathbf{C R}$ are defined, respectively, by the pseudoidentities $a b^{\omega} c=\left(a b^{\omega} c\right)^{\omega+1}$, $a^{\omega} b=\left(a^{\omega} b\right)^{\omega+1}$ and $b a^{\omega}=\left(b a^{\omega}\right)^{\omega+1}$.

Let $\mathbf{V}$ be a pseudovariety. The semigroup of ( $n$-ary) implicit operations on $\mathbf{V}$ is denoted by $\hat{F}_{n}(\mathbf{V})$ and the subsemigroup of its explicit elements is denoted by $F_{n}(\mathbf{V})$. A pseudoidentity is a formal identity $x=y$ of elements of $\hat{F}_{n}(\mathbf{S})$, where $\mathbf{S}$ denotes the pseudovariety of all finite semigroups. If $x$ and $y$ are explicit operations, $x=y$ is called an identity.

We recall the following well-known and useful observation about the pseudovarieties $\mathcal{L} \mathbf{I}$ and $\mathbf{K}$. See, for instance, [3, pp. 88-91] for a proof.

Lemma 2.1 Let $\mathbf{V}$ be one of the pseudovarieties $\mathcal{L} \mathbf{I}$ or $\mathbf{K}$.

(1) The pseudovariety $\mathbf{V}$ does not satisfy any non-trivial identity. Furthermore, if $\mathbf{V}$ satisfies a pseudoidentity $y=u$, with $u$ explicit, then $y$ and $u$ are equal.

(2) If $\mathbf{V}$ is the pseudovariety $\mathcal{L} \mathbf{I}$ (resp. $\mathbf{K}$ ) and $\mathbf{V}$ satisfies a pseudoidentity $x=y$, with $x$ and $y$ not explicit, then there exist $r, s, u, v \in \hat{F}_{n}(\mathbf{S})$, with $r$ and $s$ not explicit, such that $x=$ rus and $y=r v s$ (resp. $x=r u$ and $y=r v)$. 
The next result, due to Almeida [3, Corollary 5.6.2], is also very useful.

Lemma 2.2 If $x \in \hat{F}_{n}(\mathbf{S})$ is a non-explicit operation, then there exist $x_{1}, x_{2}, x_{3} \in \hat{F}_{n}(\mathbf{S})$ such that $x=x_{1} x_{2}^{\omega} x_{3}$.

We shall need the following result, proved by Almeida and Azevedo, which can be found in [7].

Proposition 2.3 Let $\mathbf{V}$ be a subpseudovariety of $\mathcal{D} \mathbf{R e G}$ and let $x, y \in \hat{F}_{n}(\mathbf{S})$. If $x$ and $y$ are regular when restricted to $\mathbf{V}$, then $\mathbf{V}$ satisfies $x=y$ if and only if $\mathbf{V}$ satisfies $x^{\omega}=y^{\omega}$ and $\mathbf{V} \cap \mathbf{G}$ satisfies $x=y$.

\section{The computations}

Let $\Sigma$ be a set of pseudoidentities and let $a, b, c$ and $d$ be symbols not appearing in $\Sigma$. In [11] the author considered the following pseudovarieties

$$
\begin{aligned}
\mathbf{U}_{\Sigma} & =\llbracket a^{\omega} b x c d^{\omega}=a^{\omega} b y c d^{\omega} \mid x=y \in \Sigma \rrbracket \\
\mathbf{U}_{\Sigma, l} & =\llbracket a^{\omega} b x=a^{\omega} b y \mid x=y \in \Sigma \rrbracket
\end{aligned}
$$

and showed that, if $\Sigma$ and $\Sigma^{\prime}$ are two sets of pseudoidentities defining the same pseudovariety, then $\mathbf{U}_{\Sigma}=\mathbf{U}_{\Sigma^{\prime}}$ and $\mathbf{U}_{\Sigma, l}=\mathbf{U}_{\Sigma^{\prime}, l}$. As a consequence, the author introduced the following operators on the lattice of pseudovarieties: for a pseudovariety $\mathbf{V}=\llbracket \Sigma \rrbracket$,

$$
\mathcal{U} \mathbf{V}=\mathbf{U}_{\Sigma} \quad \text { and } \quad \mathcal{U}_{l} \mathbf{V}=\mathbf{U}_{\Sigma, l} .
$$

Let us now define, for a set $\Sigma$ of pseudoidentities and symbols $a, b, c$ and $d$ not appearing in $\Sigma$,

$$
\begin{aligned}
\mathbf{U}_{\Sigma}^{\omega} & =\llbracket\left(a^{\omega} b x c d^{\omega}\right)^{\omega}=\left(a^{\omega} b y c d^{\omega}\right)^{\omega} \mid x=y \in \Sigma \rrbracket \\
\mathbf{U}_{\Sigma, l}^{\omega} & =\llbracket\left(a^{\omega} b x\right)^{\omega}=\left(a^{\omega} b y\right)^{\omega} \mid x=y \in \Sigma \rrbracket .
\end{aligned}
$$

Proposition 3.1 Let $\Sigma$ and $\Sigma^{\prime}$ be two sets of pseudoidentities defining the same pseudovariety, i.e., such that $\llbracket \Sigma \rrbracket=\llbracket \Sigma^{\prime} \rrbracket$. Then $\mathbf{U}_{\Sigma}^{\omega}=\mathbf{U}_{\Sigma^{\prime}}^{\omega}$ and $\mathbf{U}_{\Sigma, l}^{\omega}=\mathbf{U}_{\Sigma^{\prime}, l}^{\omega}$.

Proof. Using the fact that the correspondence $x \mapsto x^{\omega}$, defined on $\hat{F}_{n}(\mathbf{S})$, is continuous, the proof is a simple adaptation of the proof of [11, Proposition 3.1]. It suffices to make on that proof the obvious changes, namely the substitution of the implicit operations of the form $a^{\omega} b z c d^{\omega}$ by $\left(a^{\omega} b z c d^{\omega}\right)^{\omega}$ and the pseudovarieties of the form $\mathbf{U}_{\Phi}$ by $\mathbf{U}_{\Phi}^{\omega}$.

As a consequence of this result, we may introduce the following two operators on the lattice of pseudovarieties. For a pseudovariety $\mathbf{V}=\llbracket \Sigma \rrbracket$, we let

$$
\mathcal{U}^{\omega} \mathbf{V}=\mathbf{U}_{\Sigma}^{\omega} \quad \text { and } \quad \mathcal{U}_{l}^{\omega} \mathbf{V}=\mathbf{U}_{\Sigma, l}^{\omega} .
$$

Notice that, for pseudovarieties $\mathbf{V}$ and $\mathbf{W}$, the following properties hold:

- $\mathcal{L} \mathbf{I} \vee \mathbf{V} \subseteq \mathcal{U} \mathbf{V} \subseteq \mathcal{U}^{\omega} \mathbf{V}$ and $\mathbf{K} \vee \mathbf{V} \subseteq \mathcal{U}_{l} \mathbf{V} \subseteq \mathcal{U}_{l}^{\omega} \mathbf{V}$

- $\mathcal{L} \mathbf{I} \vee \mathbf{G} \vee \mathbf{V} \subseteq \mathcal{U}^{\omega} \mathbf{V}$ and $\mathbf{K} \vee \mathbf{G} \vee \mathbf{V} \subseteq \mathcal{U}_{l}^{\omega} \mathbf{V}$; 
- if $\mathbf{V} \subseteq \mathbf{W}$, then $\mathcal{U}^{\omega} \mathbf{V} \subseteq \mathcal{U}^{\omega} \mathbf{W}$ and $\mathcal{U}_{l}^{\omega} \mathbf{V} \subseteq \mathcal{U}_{l}^{\omega} \mathbf{W}$

Remark. We notice that, if on the definition of the operator $\mathcal{U}^{\omega}$ one would substitute the $\omega$ by an arbitrary positive integer $k$, one would obtain a new (well-defined) operator. That is, the operators $\mathcal{U}$ and $\mathcal{U}^{\omega}$ can be obtained as members of a countable family of operators $\left(\mathcal{U}^{k}\right)_{k \in \mathbb{N} \cup\{\omega\}}$, where, for a pseudovariety $\mathbf{V}=\llbracket \Sigma \rrbracket$, we let

$$
\mathcal{U}^{k} \mathbf{V}=\llbracket\left(a^{\omega} b x c d^{\omega}\right)^{k}=\left(a^{\omega} b y c d^{\omega}\right)^{k} \mid x=y \in \Sigma \rrbracket .
$$

We can now present the announced characterization of the pseudovarieties of the form $\mathcal{L} \mathbf{I} \vee \mathbf{H} \vee \mathbf{V}$, with $\mathbf{V} \subseteq(\mathbf{C R} m \mathbf{N}) \cap \mathcal{D} \mathbf{R e H}$. We denote by $\mathbf{K}_{1}\left(\right.$ resp. $\left.\mathbf{D}_{1}\right)$ the pseudovariety of left zero (resp. right zero) semigroups.

Theorem 3.2 Let $\mathbf{H}$ be a pseudovariety of groups and let $\mathbf{V}$ be a subpseudovariety of $\mathcal{L}_{l} \mathbf{C R} \cap \mathcal{L}_{r} \mathbf{C R} \cap \mathcal{D} \mathbf{R e H}$. Then

$$
\begin{aligned}
\mathcal{L} \mathbf{I} \vee \mathbf{H} \vee \mathbf{V} & =\mathcal{U}^{\omega} \mathbf{V} \cap \mathcal{L}_{l} \mathbf{C R} \cap \mathcal{L}_{r} \mathbf{C R} \cap \mathcal{D} \mathbf{R e H} \\
& =\mathcal{U}^{\omega} \mathbf{V} \cap \mathcal{L}_{l}\left(\mathbf{D}_{1} \vee \mathbf{H} \vee \mathbf{V}\right) \cap \mathcal{L}_{r}\left(\mathbf{K}_{1} \vee \mathbf{H} \vee \mathbf{V}\right) \cap \mathcal{D} \mathbf{R e H} \\
\mathbf{K} \vee \mathbf{H} \vee \mathbf{V} & =\mathcal{U}_{l}^{\omega} \mathbf{V} \cap \mathcal{L}_{l} \mathbf{C R} \cap \mathcal{L}_{r} \mathbf{C R} \cap \mathcal{D} \mathbf{R e H} \\
& =\mathcal{U}_{l}^{\omega} \mathbf{V} \cap \mathcal{L}_{l}\left(\mathbf{D}_{1} \vee \mathbf{H} \vee \mathbf{V}\right) \cap \mathcal{L}_{r}\left(\mathbf{K}_{1} \vee \mathbf{H} \vee \mathbf{V}\right) \cap \mathcal{D} \mathbf{R e H} .
\end{aligned}
$$

Proof. The other case being similar, we show the result only for $\mathcal{L} \mathbf{I}$. Let

$$
\mathbf{W}=\mathcal{U}^{\omega} \mathbf{V} \cap \mathcal{L}_{l} \mathbf{C R} \cap \mathcal{L}_{r} \mathbf{C R} \cap \mathcal{D} \mathbf{R e H} .
$$

The inclusion $\mathcal{L} \mathbf{I} \vee \mathbf{H} \vee \mathbf{V} \subseteq \mathbf{W}$ is clear. For the proof of the reverse inclusion, consider a pseudoidentity $x=y$ and suppose that $\mathcal{L} \mathbf{I} \vee \mathbf{H} \vee \mathbf{V}$ satisfies $x=y$. By Reiterman's Theorem, it suffices to prove that $\mathbf{W}$ satisfies $x=y$.

Since $\mathcal{L}$ I satisfies $x=y$, by Lemma 2.1 (1) two cases may arise: either $x$ and $y$ are the same word or $x$ and $y$ are both not explicit. In this last case, Lemma 2.1 (2) shows that we can write $x=r u s$ and $y=r v s$, for some $r, s, u, v \in \hat{F}_{n}(\mathbf{S})$ with $r$ and $s$ not explicit. Moreover, from Lemma 2.2, we can write $r=r_{1} r_{2}^{\omega} r_{3}$ and $s=s_{1} s_{2}^{\omega} s_{3}$, for some $r_{1}, r_{2}, r_{3}, s_{1}, s_{2}, s_{3} \in \hat{F}_{n}(\mathbf{S})$. Now, we notice that $\mathcal{L}_{l} \mathbf{C R} \cap \mathcal{L}_{r} \mathbf{C R}$ satisfies

$$
x=r u s=r_{1} r_{2}^{\omega} r_{3} u s_{1} s_{2}^{\omega} s_{3}=\left(r_{1} r_{2}^{\omega}\right)^{\omega+1} r_{3} u s_{1}\left(s_{2}^{\omega} s_{3}\right)^{\omega+1}=\left(r_{1} r_{2}^{\omega}\right)^{\omega} x\left(s_{2}^{\omega} s_{3}\right)^{\omega} .
$$

Analogously, $\mathcal{L}_{l} \mathbf{C R} \cap \mathcal{L}_{r} \mathbf{C R}$ satisfies $y=\left(r_{1} r_{2}^{\omega}\right)^{\omega} y\left(s_{2}^{\omega} s_{3}\right)^{\omega}$. Now since $\mathbf{V}$ satisfies $x=y$ it is clear, from its definition, that $\mathcal{U}^{\omega} \mathbf{V}$ satisfies $\left(\left(r_{1} r_{2}^{\omega}\right)^{\omega} x\left(s_{2}^{\omega} s_{3}\right)^{\omega}\right)^{\omega}=\left(\left(r_{1} r_{2}^{\omega}\right)^{\omega} y\left(s_{2}^{\omega} s_{3}\right)^{\omega}\right)^{\omega}$. Therefore, W satisfies

$$
x^{\omega}=\left(\left(r_{1} r_{2}^{\omega}\right)^{\omega} x\left(s_{2}^{\omega} s_{3}\right)^{\omega}\right)^{\omega}=\left(\left(r_{1} r_{2}^{\omega}\right)^{\omega} y\left(s_{2}^{\omega} s_{3}\right)^{\omega}\right)^{\omega}=y^{\omega} .
$$

We notice that, since $\mathbf{W} \subseteq \mathcal{L}_{l} \mathbf{C R} \cap \mathcal{L}_{r} \mathbf{C R}=\mathbf{C R} m \mathbf{N}$, an immediate consequence of Lemma 2.2 is that every non-explicit operation is a regular element when restricted to $\mathbf{W}$. Therefore, since $\mathbf{H}=\mathbf{W} \cap \mathbf{G}$ satisfies $x=y$ and $\mathbf{W} \subseteq \mathcal{D} \mathbf{R e G}$, we deduce from Proposition 2.3 that $\mathbf{W}$ satisfies $x=y$.

To prove the second equality concerning $\mathcal{L} \mathbf{I}$ let us note that the following relations are valid

$$
\mathcal{L} \mathbf{I} \vee \mathbf{H} \vee \mathbf{V} \subseteq \mathcal{L}_{l}\left(\mathbf{D}_{1} \vee \mathbf{H} \vee \mathbf{V}\right) \subseteq \mathcal{L}_{l}\left(\mathcal{L}_{l} \mathbf{C R}\right)=\mathcal{L}_{l} \mathbf{C R}
$$

Analogously, $\mathcal{L} \mathbf{I} \vee \mathbf{H} \vee \mathbf{V} \subseteq \mathcal{L}_{r}\left(\mathbf{K}_{1} \vee \mathbf{H} \vee \mathbf{V}\right) \subseteq \mathcal{L}_{r} \mathbf{C R}$. Now, these inclusions and the first equality concerning $\mathcal{L} \mathbf{I}$ imply the second one. 


\section{Conclusion and open questions}

Theorem 3.2 presents a description of the pseudovarieties of the form $\mathcal{L} \mathbf{I} \vee \mathbf{H} \vee \mathbf{V}$, with $\mathbf{V} \subseteq(\mathbf{C R} m \mathbf{N}) \cap \mathcal{D} \mathbf{R e H}$. We must refer that these calculations were obtained in [11] but under a different form. In fact [11, Theorem 4.1] presents a description of the pseudovarieties $\mathcal{L} \mathbf{I} \vee \mathbf{W}$, with $\mathbf{W} \subseteq \mathbf{C R} m \mathbf{N}$. Thus, in particular when $\mathbf{W}=\mathbf{H} \vee \mathbf{V}$, [11, Theorem 4.1] presents a characterization of $\mathcal{L} \mathbf{I} \vee \mathbf{H} \vee \mathbf{V}$, with $\mathbf{V} \subseteq(\mathbf{C R} m \mathbf{N}) \cap \mathcal{D} \mathbf{R e H}$. However, this characterization is obtained in terms of the pseudovariety $\mathcal{U} \mathbf{W}=\mathcal{U}(\mathbf{H} \vee$ $\mathbf{V}$ ), and so in terms of a set of pseudoidentities defining $\mathbf{H} \vee \mathbf{V}$. Therefore Theorem 3.2 above gives effectively a new description of the joins $\mathcal{L} \mathbf{I} \vee \mathbf{H} \vee \mathbf{V}$, since it presents them in terms of the pseudovariety $\mathcal{U}^{\omega} \mathbf{V}$, and so in terms of a set of pseudoidentities defining $\mathbf{V}$. Therefore, the operator $\mathcal{U}^{\omega}$ seams to be more appropriate to characterize the pseudovarieties $\mathcal{L} \mathbf{I} \vee \mathbf{H} \vee \mathbf{V}$.

As to the pseudovarieties not contained in $(\mathbf{C R}: \mathbf{N}) \cap \mathcal{D} \mathbf{R e H}$, some calculations of the form $\mathcal{L} \mathbf{I} \vee \mathbf{H} \vee \mathbf{V}$ are known from [11], in terms of the operator $\mathcal{U}$. For instance, as a consequence of the considerations of Section 5 in [11], we deduce the equality

$$
\mathcal{L} \mathbf{I} \vee \mathbf{G} \vee \mathbf{J}=\mathcal{U}(\mathbf{G} \vee \mathbf{J})
$$

Trotter and Volkov [16] have shown that the pseudovariety $\mathbf{G} \vee \mathbf{J}$ is not finitely based, while Steinberg [15] has proved that $\mathbf{G} \vee \mathbf{J}$ is definable by a recursive set of pseudoidentities. So, $\mathcal{L} \mathbf{I} \vee \mathbf{G} \vee \mathbf{J}$ is also definable by a recursive set of pseudoidentities. We propose the following question, in terms of the operator $\mathcal{U}^{\omega}$.

Problem 4.1 Does the equality $\mathcal{L} \mathbf{I} \vee \mathbf{G} \vee \mathbf{J}=\mathcal{U}^{\omega} \mathbf{J} \cap \mathcal{D} \mathbf{R e G}$ hold?

The positive answer to this question would give a finite basis for $\mathcal{L} \mathbf{I} \vee \mathbf{G} \vee \mathbf{J}$.

\section{References}

[1] D. Albert, R. Baldinger and J. Rhodes, Undecidability of the identity problem for finite semigroups, J. Symbolic Logic 57 (1992) 179-192.

[2] J. Almeida, Some pseudovariety joins involving the pseudovariety of finite groups, Semigroup Forum 37 (1988) 53-57.

[3] J. Almeida, Finite Semigroups and Universal Algebra, World Scientific, Singapore, 1994.

[4] J. Almeida and A. Azevedo, The join of the pseudovarieties of $\mathcal{R}$-trivial and $\mathcal{L}$ trivial semigroups, J. Pure and Applied Algebra 60 (1989) 129-137.

[5] J. Almeida, A. Azevedo and M. Zeitoun, Pseudovariety joins involving $\mathcal{J}$-trivial semigroups and completely regular semigroups, Int. J. Algebra and Computation 9 (1999) 99-112.

[6] J. Almeida and P. Weil, Reduced factorizations in free profinite groups and join decompositions of pseudovarieties, Int. J. Algebra and Computation 4 (1994) 375403. 
[7] A. Azevedo, Operations preserving homomorphisms on the class of finite semigroups DS, in J. Almeida (Ed.), Actas II Encontro de Algebristas Portugueses, Universidade do Porto, Porto (1987) 33-43.

[8] A. Azevedo, The join of the pseudovariety $\mathbf{J}$ with permutative pseudovarieties, in J. Almeida and al (Eds.), Lattices, Semigroups and Universal Algebra, Plenum, New York (1990) 1-11.

[9] A. Azevedo and M. Zeitoun, Three examples of join computations, Semigroup Forum 57 (1998) 249-277.

[10] J. Costa, Free profinite semigroups over some classes of semigroups locally in DG, Int. J. Algebra and Computation 10 (2000) 491-537.

[11] J. Costa, Some pseudovariety joins involving locally trivial semigroups, Semigroup Forum, to appear.

[12] S. Eilenberg, Automata, Languages and Machines, vol. B, Academic Press, New York, 1976.

[13] J.-E. Pin, Varieties of Formal Languages, Plenum, New York and North Oxford, London, 1986.

[14] B. Steinberg, On pointlike sets and joins of pseudovarieties, Int. J. Algebra and Computation 8 (1998) 203-231.

[15] B. Steinberg, On algorithmic problems for joins of pseudovarieties, Semigroup Forum 62 (2001) 1-40.

[16] P. Trotter and M. Volkov, The finite basis problem in the pseudovariety joins of aperiodic semigroups with groups, Semigroup Forum 52 (1996) 83-91.

[17] M. Zeitoun, On the decidability of the membership problem of the pseudovariety $\mathbf{J} \vee \mathbf{B}$, Int. J. Algebra and Computation 5 (1995) 47-64.

[18] M. Zeitoun, On the join of two pseudovarieties, in J. Almeida and al (Eds.), Semigroups, Automata and Languages, World Scientific, Singapore (1996) 281-288. 